\title{
Prognosis of Acute Kidney Injury and Hepatorenal Syndrome in Patients with Cirrhosis: A Prospective Cohort Study
}

\author{
Andrew S. Allegretti, ${ }^{1}$ Guillermo Ortiz, ${ }^{1}$ Julia Wenger, ${ }^{1}$ Joseph J. Deferio, ${ }^{1}$ \\ Joshua Wibecan, ${ }^{1}$ Sahir Kalim, ${ }^{1}$ Hector Tamez, ${ }^{2}$ Raymond T. Chung, ${ }^{3}$ \\ S. Ananth Karumanchi, ${ }^{4}$ and Ravi I. Thadhani ${ }^{1}$ \\ ${ }^{1}$ Division of Nephrology, Department of Medicine, Massachusetts General Hospital, Boston, MA 02114, USA \\ ${ }^{2}$ Division of Cardiology, Department of Medicine, Beth Israel Deaconess Medical Center, Boston, MA 02114, USA \\ ${ }^{3}$ Liver Center and Gastrointestinal Division, Department of Medicine, Massachusetts General Hospital, Boston, MA 02114, USA \\ ${ }^{4}$ Division of Nephrology, Department of Medicine, Beth Israel Deaconess Medical Center, Boston, MA 02215, USA
}

Correspondence should be addressed to Andrew S. Allegretti; aallegretti@partners.org

Received 22 April 2015; Revised 28 June 2015; Accepted 6 July 2015

Academic Editor: Suresh C. Tiwari

Copyright (C) 2015 Andrew S. Allegretti et al. This is an open access article distributed under the Creative Commons Attribution License, which permits unrestricted use, distribution, and reproduction in any medium, provided the original work is properly cited.

\begin{abstract}
Background/Aims. Acute kidney injury is a common problem for patients with cirrhosis and is associated with poor survival. We aimed to examine the association between type of acute kidney injury and 90-day mortality. Methods. Prospective cohort study at a major US liver transplant center. A nephrologist's review of the urinary sediment was used in conjunction with the 2007 Ascites Club Criteria to stratify acute kidney injury into four groups: prerenal azotemia, hepatorenal syndrome, acute tubular necrosis, or other. Results. 120 participants with cirrhosis and acute kidney injury were analyzed. Ninety-day mortality was 14/40 (35\%) with prerenal azotemia, 20/35 (57\%) with hepatorenal syndrome, 21/36 (58\%) with acute tubular necrosis, and 1/9 (11\%) with other $(p=0.04$ overall). Mortality was the same in hepatorenal syndrome compared to acute tubular necrosis $(p=0.99)$. Mortality was lower in prerenal azotemia compared to hepatorenal syndrome $(p=0.05)$ and acute tubular necrosis $(p=0.04)$. Ten participants $(22 \%)$ were reclassified from hepatorenal syndrome to acute tubular necrosis because of granular casts on urinary sediment. Conclusions. Hepatorenal syndrome and acute tubular necrosis result in similar 90-day mortality. Review of urinary sediment may add important diagnostic information to this population. Multicenter studies are needed to validate these findings and better guide management.
\end{abstract}

\section{Introduction}

Acute kidney injury (AKI) is a common and life-threatening problem for patients with cirrhosis [1-3]. The differential diagnosis for AKI in this population is large. The most common etiologies are prerenal azotemia (PRA), acute tubular necrosis (ATN), and hepatorenal syndrome (HRS), but other causes such as glomerulonephritis, medication toxicity, and abdominal compartment syndrome from tense ascites occur as well $[3,4]$. Regardless of etiology, AKI is associated with reduced survival [5-7]. Measures of renal function (i.e., serum creatinine) are factored prominently into prognostic scores such as the Model for End-Stage Liver Disease (MELD) and Chronic Liver Failure-Sequential Organ Failure Assessment (CLIF-SOFA) and have major implications for liver transplant allocation [8-10].
One cause of AKI unique to liver disease is HRS. Hepatorenal syndrome is thought to be due to splanchnic vasodilation causing hormonal imbalances that ultimately result in renal vasoconstriction and impaired renal function $[4,11]$. Several studies suggest that HRS is associated with the highest mortality of all types of AKI [12-14]. As such, a diagnosis of HRS-related AKI is felt to have greater clinical significance over other types of AKI. Despite this, there is no definitive test for HRS, which remains a challenging diagnosis for clinicians.

Over the last several years, consensus guidelines have evolved to aid in the diagnosis of AKI in cirrhosis. The diagnostic criteria for HRS have been updated several times, most recently in $2007[14,15]$. The definition of AKI itself has been debated as well, as some of the older definitions of AKI that used a doubling of serum creatinine or a preset threshold 
value had limited sensitivity to detect AKI in patients with cirrhosis $[16,17]$. Recent studies support using the Acute Kidney Injury Network (AKIN) definition of AKI, which correlates closely with mortality in cirrhosis [17-23]. We sought to evaluate whether the type of AKI influenced the 90-day mortality of hospitalized patients with cirrhosis. We hypothesized that those with HRS would have the worst 90-day mortality.

\section{Methods}

2.1. Study Population and Setting. Between January 2013 and December 2014, consenting adult patients (age 18 years or older) who were hospitalized at Massachusetts General Hospital with cirrhosis and AKI (see "Definitions") were enrolled in this prospective study. Massachusetts General Hospital is a 1008-bed academic tertiary care center with an active liver transplant program. Potential participants were excluded from this study if they previously received a renal transplant, if they were on renal replacement therapy at the time of admission, or if they were pregnant or nursing. Participants were followed during their inpatient admission and subsequently as outpatients after discharge.

\subsection{Definitions}

2.2.1. Cirrhosis. The diagnosis of cirrhosis was based on clinical evaluation by a hepatologist using laboratory values, liver imaging, endoscopy, and (when available) liver biopsy.

2.2.2. Acute Kidney Injury. The AKIN criteria were used to diagnose AKI, which required an absolute increase in serum creatinine of $0.3 \mathrm{mg} / \mathrm{dL}(26.4 \mu \mathrm{mol} / \mathrm{L})$ above baseline or an increase of serum creatinine to $150 \%$ of baseline within 48 hours [21]. The AKIN criterion for decline in urine output was not used in the initial diagnosis of AKI as it was felt to be unreliable in patients with ascites and without a bladder catheter.

2.2.3. Classification of AKI. Participants were classified as having one of four types of AKI: (1) prerenal azotemia (PRA), (2) hepatorenal syndrome (HRS), (3) acute tubular necrosis (ATN), and (4) other causes. Participants were classified as having HRS based on the 2007 Ascites Club Criteria [14]. These include the following: (1) presence of cirrhosis and ascites, (2) serum creatinine greater than $1.5 \mathrm{mg} / \mathrm{dL}$, (3) failure of improvement in serum creatinine below $1.5 \mathrm{mg} / \mathrm{dL}$ after administration of albumin and withdrawal of diuretics for 48 hours, (4) absence of nephrotoxic drugs, (5) absence of shock, and (6) absence of parenchymal renal disease. Parenchymal renal disease was defined as presence of abnormal kidneys on ultrasound, $>500 \mathrm{mg}$ proteinuria per day, presence of $>50$ RBCs per high-powered field of urinary sediment, or presence of granular casts on a nephrologist's review of the urinary sediment. Participants were classified as having PRA if they presented with AKI, a clinical history consistent with a prerenal state (such as bleeding or GI fluid losses), and their serum creatinine improved following the administration of volume and withdrawal of diuretics. Participants were diagnosed with ATN if they failed to meet criteria for PRA and HRS and had a clinical history consistent with tubular/parenchymal kidney injury. Participants were diagnosed with "other" causes of AKI if they had evidence of another process on serology or renal biopsy, such as glomerulonephritis.

Diagnoses of AKI were reviewed and confirmed independently by two study investigators. If a discrepancy in diagnosis was found, a third investigator reviewed the medical record and provided a tie-breaking diagnosis.

2.3. Data Collection and Management. All data was registered as part of the routine clinical care and collected from review of the electronic medical record. This included demographics, laboratory findings, radiology, procedural findings (including microscopic examination of urine sediment by a nephrologist), medical history, and medications. MELD and CLIFSOFA scores were taken from the time of admission. The reference laboratory at the study site reported the lowest cutoff of urine sodium as "less than $10 \mathrm{mmol} / \mathrm{L}$," so this variable was dichotomized for analysis at this value. All values reported are taken from the time of enrollment unless otherwise noted. Study data were collected and managed using REDCap electronic data capture tools hosted at the Harvard Clinical and Translational Science Center [24]. Each participant also provided serum, plasma, and urine samples on enrollment and on days 5 and 30 after enrollment (as available) to create a biorepository for future evaluation of markers of kidney and liver injury.

Participants were treated as per local standard of care by the managing internists, hepatologists, and nephrologists on service. Members of the study team did not intervene in patient care. Management of AKI was done using guidelines and evidenced-based medicine at the discretion of treating clinicians, including (1) withdrawal of potential offending agents, such as diuretics, (2) empiric and culture based treatment of infections, (3) administration of volume, (4) reversal of underlying insults, such as endoscopic treatment of bleeding, (5) treatment of suspected HRS using albumin and midodrine/octreotide or vasopressors, and (6) use of intermittent hemodialysis or continuous venovenous hemofiltration for AKI refractory to medical management. Participants diagnosed with spontaneous bacterial peritonitis were given $1.5 \mathrm{~g} / \mathrm{kg}$ albumin on day of diagnosis and $1 \mathrm{~g} / \mathrm{kg}$ albumin 48 hours later along with antibiotics [25].

2.4. Statistical Analysis. Demographic and clinical characteristics of participants are presented as medians (quartile 1, quartile 3) or number (percentage) and compared between the four subgroups of AKI by one-way analysis of variance tests or Fisher's exact tests. These variables were also compared between those alive and those who died at 90 days using Mann-Whitney $U$ tests for continuous variables and Fisher's exact test for categorical variables. Additional outcomes (need for dialysis, recovery from dialysis, creatinine at 90 days, and liver transplantation after enrollment) were analyzed by type of AKI in a similar fashion. Pairwise testing was also performed to compare outcomes between participants with HRS versus ATN. 
Differences in the primary outcome of death by 90 days by type of AKI were visualized using a Kaplan-Meier curve and compared using a log-rank test. Cox proportional hazard models were used to create a multivariable model to predict death by 90 days by type of AKI. The subgroup of "other causes" of AKI was not analyzed in the multivariable model to better highlight the three subgroups affected by altered hemodynamics (PRA, HRS, and ATN). Because of sample size limitations, two prespecified models were selected for analysis using type of AKI, age, and either MELD or CLIF-SOFA score as predictors. The assumption of proportional hazards was tested for all models. Results of Cox proportional hazard models are summarized with hazard ratios and Wald asymptotic $95 \%$ confidence intervals. Four sensitivity analyses were performed: (1) excluding four participants who had previously received a liver transplant, (2) using a composite endpoint of death or liver transplant by 90 days, (3) reclassifying 10 participants with granular casts on sediment as having HRS if all other criteria were met, and (4) including infection as a subgroup of AKI (AKI with infection compared to three subgroups [PRA, HRS, and HRS] without infection). SAS version 9.4 (Gary, NC) was used for all analyses. Two-tailed $p$ values $<$ 0.05 were considered to indicate statistical significance.

2.5. Ethics Statement. This study was approved by the site's institutional review board and abides by the guidelines set forth by the Declaration of Helsinki. No donor organs were obtained from executed prisoners or other institutionalized persons at this liver transplant center. All participants (or their health care designee) provided written informed consent.

\section{Results}

3.1. General Demographics. One hundred twenty participants with cirrhosis and AKI were analyzed in this study. Forty (33\%) had PRA, 35 (29\%) had HRS, 36 (30\%) had ATN, and $9(8 \%)$ had other causes of AKI (Figure 1). Of the other causes of AKI, 5 (56\%) had evidence of glomerulonephritis. Ten participants who met all inclusion criteria for HRS (22\%) were reclassified to acute tubular necrosis because of the presence of granular casts on urinary sediment.

Median (quartile 1, quartile 3 ) age of the entire cohort was $58(50,65)$ years. The majority of participants were male $(71 \%)$, white race $(93 \%)$, and of non-Hispanic ethnicity (87\%). Median length of hospital admission was $16(9,24)$ days. Median time from admission to enrollment was $4(3,10)$ days. Median MELD score was $24(18,30)$ and median CLIFSOFA score was $9(6,10)$. The most common etiologies of cirrhosis were alcoholic (30\%), multifactorial (27\%), and hepatitis C (20\%). Eleven participants (9\%) had stage I AKI, 23 participants (19\%) had stage II AKI, and 86 participants (72\%) had stage III AKI. Ninety-four participants (78\%) received nephrology consultation. Thirty-eight participants (32\%) required renal replacement therapy. Twenty-one participants $(18 \%)$ went on to receive liver transplantation. Fortynine participants (41\%) received vasopressors while being hospitalized in the intensive care unit. Thirty-one participants with HRS (89\%) were treated with midodrine and

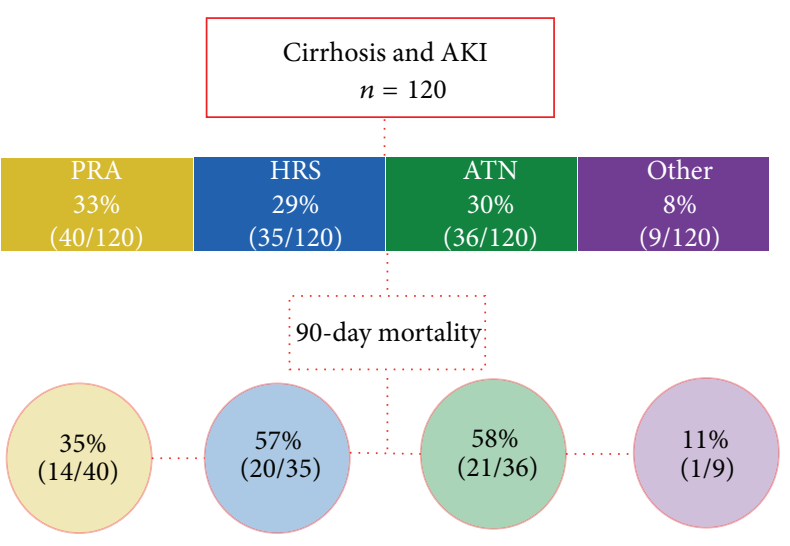

FIGURE 1: Distribution of participants and 90-day mortality. AKI (acute kidney injury), PRA (prerenal azotemia), HRS (hepatorenal syndrome), and ATN (acute tubular necrosis).

octreotide. Characteristics of all participants by type of AKI are presented in Table 1 .

Across the entire cohort, 56 (47\%) participants died by day 90 after enrollment. Participants who died had a significantly higher MELD score, CLIF-SOFA score, serum creatinine on enrollment, peak creatinine during their admission, INR, and total bilirubin and had a lower urine output and mean arterial pressure. Participants who died were more likely to receive nephrology consultation, receive dialysis, receive intravenous albumin, or be treated with midodrine, octreotide, or intravenous vasopressors (Table 2).

Among those with HRS, 13/15 (87\%) who survived and $18 / 20(90 \%)$ who died were treated with midodrine and octreotide $(p=1.00)$. Intravenous vasopressors were given to $6 / 15(40 \%)$ who survived and 10/20 (50\%) who died $(p=$ $0.73)$.

3.2. Outcomes by Type of AKI. The primary and secondary outcomes were analyzed across all four types of AKI (PRA, HRS, ATN, and other) and in pairwise analysis between those with HRS and ATN (Table 3 ). There was a significant difference in death by 90 days across all four subgroups $(p=0.02)$ but not between HRS and ATN (20/35 [57\%] versus 21/36 [58\%]; $p=1.00)$. There was no significant difference in the need for dialysis $(p=0.13)$, recovery from dialysis (among survivors to 90 days; $p=0.16$ ), and new liver transplant $(p=0.44)$. Among those who were alive and did not require dialysis, participants with HRS had a higher serum creatinine at 90 days than those with ATN $(1.5[1.2,2.0] \mathrm{mg} / \mathrm{dL}$ versus 1.0 $[0.8,1.5] \mathrm{mg} / \mathrm{dL} ; p=0.01$ ).

Overall, MELD score was significantly different across all four groups for all participants ( $p=0.04)$, but not between HRS and ATN (24 [21,31] versus $26[20,34] ; p=0.67)$. Among those who died, there was no significant difference in MELD score across all four groups $(p=0.90)$. CLIF-SOFA score was significantly different across all four groups for all participants $(p=0.01)$, but not between HRS and ATN (9 $[8,10]$ versus $10[7,12] ; p=0.17)$. Similarly, there was no significant difference in CLIF-SOFA scores among participants 
TABLE 1: Demographics and clinical characteristics.

\begin{tabular}{|c|c|c|c|c|c|}
\hline & $\begin{array}{c}\text { PRA } \\
(n=40)\end{array}$ & $\begin{array}{c}\text { HRS } \\
(n=35)\end{array}$ & $\begin{array}{c}\text { ATN } \\
(n=36)\end{array}$ & $\begin{array}{l}\text { Other } \\
(n=9)\end{array}$ & $p$ value \\
\hline Age (years) & $58(49.5,66)$ & $57(49,65)$ & $60(51,63)$ & $57(51,62)$ & 0.80 \\
\hline Male sex (\%) & $26(65 \%)$ & $25(71 \%)$ & $26(72 \%)$ & $8(89 \%)$ & 0.60 \\
\hline White race $(\%)$ & $34(85 \%)$ & $34(97 \%)$ & $34(94 \%)$ & $9(100 \%)$ & 0.49 \\
\hline Non-Hispanic ethnicity (\%) & $31(78 \%)$ & $30(86 \%)$ & $34(94 \%)$ & $9(100 \%)$ & 0.23 \\
\hline Body mass index $\left(\mathrm{kg} / \mathrm{m}^{2}\right)$ & $28.8(26.5,34.6)$ & $28.3(23.7,32.0)$ & $27.4(22.5,32.8)$ & $26.9(25.1,31.0)$ & 0.38 \\
\hline Presence of infection (\%) & $15(38 \%)$ & $11(31 \%)$ & $18(50 \%)$ & $1(11 \%)$ & 0.14 \\
\hline \multicolumn{6}{|l|}{ Comorbidities (\%) } \\
\hline Diabetes mellitus & $11(29 \%)$ & $10(29 \%)$ & $11(31 \%)$ & $4(8 \%)$ & 0.81 \\
\hline Chronic kidney disease & $7(18 \%)$ & $14(41 \%)$ & $10(28 \%)$ & $7(78 \%)$ & 0.01 \\
\hline Cardiovascular disease & $8(20 \%)$ & $6(17 \%)$ & $7(19 \%)$ & $3(33 \%)$ & 0.73 \\
\hline Hypertension & $17(43 \%)$ & $14(40 \%)$ & $14(40 \%)$ & $3(33 \%)$ & 0.99 \\
\hline Etiology of cirrhosis (\%) & & & & & 0.24 \\
\hline Hepatitis C & $11(28 \%)$ & $4(11 \%)$ & $7(19 \%)$ & $2(22 \%)$ & \\
\hline Alcohol & $12(30 \%)$ & $12(35 \%)$ & $12(33 \%)$ & $0(0 \%)$ & \\
\hline Nonalcoholic steatohepatitis & $3(8 \%)$ & $4(11 \%)$ & $5(14 \%)$ & $1(11 \%)$ & \\
\hline Multifactorial & $12(30 \%)$ & $11(31 \%)$ & $6(17 \%)$ & $3(33 \%)$ & \\
\hline Other & $2(5 \%)$ & $4(11 \%)$ & $6(17 \%)$ & $3(33 \%)$ & \\
\hline \multicolumn{6}{|l|}{ Prior complications of cirrhosis (\%) } \\
\hline Ascites requiring prior paracentesis & $14(36 \%)$ & $27(82 \%)$ & $12(33 \%)$ & $4(44 \%)$ & $<0.001$ \\
\hline Encephalopathy & $14(35 \%)$ & $20(57 \%)$ & $12(33 \%)$ & $3(33 \%)$ & 0.15 \\
\hline Gastrointestinal bleeding & $7(18 \%)$ & $8(23 \%)$ & $6(17 \%)$ & $0(0 \%)$ & 0.52 \\
\hline Spontaneous bacterial peritonitis & $4(10 \%)$ & $7(20 \%)$ & $3(8 \%)$ & $0(0 \%)$ & 0.35 \\
\hline Portosystemic shunt & $3(8 \%)$ & $2(6 \%)$ & $4(11 \%)$ & $2(22 \%)$ & 0.42 \\
\hline Prior liver transplantation (\%) & $2(5 \%)$ & $1(3 \%)$ & $0(0 \%)$ & $1(11 \%)$ & 0.57 \\
\hline MELD score (admission) & $19(17,28)$ & $24(21,31)$ & $26(20,34)$ & $20(18,21)$ & 0.04 \\
\hline CLIF-SOFA score (admission) & $8(6,9)$ & $9(8,10)$ & $10(7,12)$ & $6(5,7)$ & 0.01 \\
\hline Nephrologist consulted (\%) & $23(58 \%)$ & $31(89 \%)$ & $33(92 \%)$ & $6(67 \%)$ & $<0.001$ \\
\hline AKI stage I or II (\%)/stage III (\%) & $15(38 \%) / 25(63 \%)$ & $9(26 \%) / 26(75 \%)$ & $7(19 \%) / 29(81 \%)$ & $3(33 \%) / 6(67 \%)$ & 0.34 \\
\hline \multicolumn{6}{|l|}{ Medications received (\%) } \\
\hline Intravenous albumin & $28(70 \%)$ & $34(97 \%)^{*}$ & $29(81 \%)$ & $4(44 \%)$ & $<0.001$ \\
\hline Midodrine & $15(38 \%)$ & $31(89 \%)$ & $25(69 \%)$ & $2(22 \%)$ & $<0.001$ \\
\hline Octreotide & $21(53 \%)$ & $31(89 \%)$ & $24(67 \%)$ & $1(11 \%)$ & $<0.001$ \\
\hline Intravenous vasopressor & $13(33 \%)$ & $15(43 \%)$ & $19(53 \%)$ & $2(22 \%)$ & 0.21 \\
\hline \multicolumn{6}{|l|}{ Laboratory values/vital signs } \\
\hline Mean arterial pressure $(\mathrm{mmHg})$ & $78(73,85)$ & $74(69,80)$ & $73(68,80)$ & $86(79,88)$ & 0.07 \\
\hline Urine output (mL/24 hours) ${ }^{* *}$ & $763(475,1125)$ & $488(325,750)$ & $625(300,1095)$ & $873(525,1450)$ & 0.23 \\
\hline Enrollment creatinine (mg/dL) & $1.4(1.2,1.8)$ & $2.7(2.0,3.0)$ & $2.3(1.9,3.6)$ & $2.3(2.0,3.7)$ & $<0.001$ \\
\hline Peak creatinine $(\mathrm{mg} / \mathrm{dL})$ & $1.9(1.6,2.6)$ & $3.4(2.7,4.7)$ & $3.5(2.5,6.8)$ & $4.1(2.3,4.5)$ & $<0.001$ \\
\hline Sodium $(\mathrm{mEq} / \mathrm{L})$ & $133(130,137)$ & $131(128,136)$ & $136(132,141)$ & $136(129,140)$ & 0.10 \\
\hline White blood count (K/uL) & $7.2(4.3,10.6)$ & $7.0(4.8,10.6)$ & $9.1(5.5,15.7)$ & $6.4(4.1,8.8)$ & 0.10 \\
\hline Hemoglobin $(\mathrm{g} / \mathrm{dL})$ & $8.3(8.0,9.5)$ & $9.0(7.9,10.0)$ & $8.6(7.9,9.4)$ & $8.9(8.4,11.1)$ & 0.44 \\
\hline Platelets (K/uL) & $89(57,124)$ & $77(58,101)$ & $64(47,125)$ & $93(77,143)$ & 0.50 \\
\hline Albumin $(\mathrm{g} / \mathrm{dL})$ & $2.9(2.5,3.6)$ & $3.5(3.2,3.7)$ & $2.8(2.7,3.2)$ & $2.8(2.5,4.1)$ & 0.01 \\
\hline International normalized ratio (INR) & $1.7(1.3,2.0)$ & $1.8(1.5,2.1)$ & $1.9(1.5,2.3)$ & $1.4(1.2,1.5)$ & 0.01 \\
\hline Total bilirubin (mg/dL) & $3.8(1.7,8.2)$ & $5.0(2.2,11.0)$ & $9.6(2.5,22.2)$ & $2.0(1.1,3.8)$ & 0.06 \\
\hline Urine sodium $<10 \mathrm{mmol} / \mathrm{L}(\%)^{* * *}$ & $9(43 \%)$ & $21(75 \%)$ & $5(26 \%)$ & $2(33 \%)$ & $<0.001$ \\
\hline
\end{tabular}

All values were taken at time of study enrollment unless otherwise noted. Continuous variables presented as median (quartile 1, quartile 3 ).

PRA: prerenal azotemia, HRS: hepatorenal syndrome, and ATN: acute tubular necrosis.

* One participant received red blood cell transfusion instead of albumin.

${ }^{* *} n=36$ for PRA, $n=34$ for HRS, $n=30$ for ATN, and $n=8$ for other.

*** $n=21$ for PRA, $n=28$ for HRS, $n=19$ for ATN, and $n=6$ for other 
TABLE 2: Relationship of variables to death at 90 days for all participants* .

\begin{tabular}{|c|c|c|c|}
\hline & $\begin{array}{c}\text { Alive } \\
(n=64)\end{array}$ & $\begin{array}{c}\text { Died } \\
(n=56)\end{array}$ & $p$ value \\
\hline Age (years) & $58(48,64)$ & $59(53,66)$ & 0.12 \\
\hline Male sex (\%) & $43(69 \%)$ & $41(73 \%)$ & 0.69 \\
\hline White race $(\%)$ & $58(91 \%)$ & $53(95 \%)$ & 0.44 \\
\hline Non-Hispanic ethnicity (\%) & $53(82 \%)$ & $51(91 \%)$ & 0.34 \\
\hline Body mass index $\left(\mathrm{kg} / \mathrm{m}^{2}\right)$ & $27.2(23.6,33.2)$ & $29.0(25.5,32.2)$ & 0.47 \\
\hline Presence of infection (\%) & $20(31 \%)$ & $25(45 \%)$ & 0.14 \\
\hline \multicolumn{4}{|l|}{ Other medical problems (\%) } \\
\hline Diabetes mellitus & $19(31 \%)$ & $17(31 \%)$ & 1.00 \\
\hline Chronic kidney disease & $23(37 \%)$ & $15(27 \%)$ & 0.33 \\
\hline Cardiovascular disease & $14(22 \%)$ & $10(18 \%)$ & 0.65 \\
\hline Hypertension & $27(43 \%)$ & $21(38 \%)$ & 0.58 \\
\hline Etiology of cirrhosis ${ }^{* *}$ & - & - & 0.46 \\
\hline \multicolumn{4}{|l|}{ Prior complications of cirrhosis (\%) } \\
\hline Any ascites & $41(64 \%)$ & $33(59 \%)$ & 0.58 \\
\hline Ascites requiring paracentesis & $28(45 \%)$ & $29(53 \%)$ & 0.46 \\
\hline Encephalopathy & $27(42 \%)$ & $22(39 \%)$ & 0.85 \\
\hline Gastrointestinal bleeding & $8(13 \%)$ & $13(23 \%)$ & 0.15 \\
\hline Spontaneous bacterial peritonitis & $8(13 \%)$ & $6(11 \%)$ & 0.78 \\
\hline Portosystemic shunt & $7(11 \%)$ & $4(8 \%)$ & 0.54 \\
\hline MELD score (admission) & $23(18,29)$ & $29(23,36)$ & $<0.001$ \\
\hline CLIF-SOFA score (admission) & $8(5,10)$ & $9(8,11)$ & 0.01 \\
\hline Nephrologist consulted (\%) & $42(66 \%)$ & $51(91 \%)$ & $<0.001$ \\
\hline AKI stage I or II (\%)/stage III (\%) & $23(36 \%) / 41(64 \%)$ & $11(20 \%) / 45(80 \%)$ & 0.07 \\
\hline Required dialysis (\%) & $14(22 \%)$ & $24(43 \%)$ & 0.02 \\
\hline \multicolumn{4}{|l|}{ Medications received (\%) } \\
\hline Intravenous albumin & $45(70 \%)$ & $50(89 \%)$ & 0.01 \\
\hline Midodrine & $28(44 \%)$ & $45(80 \%)$ & $<0.001$ \\
\hline Octreotide & $32(50 \%)$ & $45(80 \%)$ & $<0.001$ \\
\hline Intravenous vasopressor & $20(31 \%)$ & $30(54 \%)$ & 0.02 \\
\hline \multicolumn{4}{|l|}{ Laboratory values/vital signs } \\
\hline Mean arterial pressure $(\mathrm{mmHg})$ & $78(71,84)$ & $74(68,80)$ & 0.03 \\
\hline Urine output (mL/24 hours) $)^{* * *}$ & $850(500,1200)$ & $450(275,750)$ & $<0.001$ \\
\hline Enrollment creatinine (mg/dL) & $1.8(1.3,2.4)$ & $2.6(1.9,3.5)$ & $<0.001$ \\
\hline Peak creatinine $(\mathrm{mg} / \mathrm{dL})$ & $2.3(1.8,3.9)$ & $3.4(2.8,4.7)$ & $<0.001$ \\
\hline Sodium (mEq/L) & $133(129,137)$ & $134(130,139)$ & 0.41 \\
\hline White blood count (K/uL) & $10.1(6.9,16.9)$ & $8.2(5.3,13.5)$ & 0.09 \\
\hline Hemoglobin $(\mathrm{g} / \mathrm{dL})$ & $8.3(7.8,9.5)$ & $8.9(8.1,10.1)$ & 0.09 \\
\hline Platelets (K/uL) & $88(59,124)$ & $72(48,104)$ & 0.20 \\
\hline Albumin $(\mathrm{g} / \mathrm{dL})$ & $2.9(2.5,3.5)$ & $3.3(2.8,3.6)$ & 0.06 \\
\hline International normalized ratio (INR) & $1.6(1.3,2.0)$ & $1.9(1.5,2.1)$ & 0.02 \\
\hline Total bilirubin (mg/dL) & $3.7(1.5,8.7)$ & $7.1(3.0,19.9)$ & 0.001 \\
\hline Urine sodium $<10 \mathrm{mmol} / \mathrm{L}(\%)^{* * * *}$ & $15(39 \%)$ & $22(61 \%)$ & 0.10 \\
\hline
\end{tabular}

All values were taken at time of study enrollment unless otherwise noted. Continuous variables presented as median (quartile 1, quartile 3 ).

AKI: acute kidney injury.

* Three participants who were lost to follow-up were included in the alive category.

** Subcategories the same as Table 1 (hepatitis C, alcohol, nonalcoholic steatohepatitis, multifactorial, other).

${ }^{* * *} n=108$ total.

${ }^{* * * *} n=74$ total. 
TABLE 3: Outcomes and variables by type of acute kidney injury.

\begin{tabular}{|c|c|c|c|c|c|c|}
\hline & $\begin{array}{c}\text { PRA } \\
n=40\end{array}$ & $\begin{array}{c}\text { HRS } \\
n=35\end{array}$ & $\begin{array}{c}\text { ATN } \\
n=36\end{array}$ & $\begin{array}{l}\text { Other } \\
n=9\end{array}$ & $\begin{array}{l}p \text { value } \\
\text { (overall) }\end{array}$ & $\begin{array}{c}p \text { value } \\
\text { (HRS versus ATN) }\end{array}$ \\
\hline Death by 90 days (\%) & $14(35 \%)$ & $20(57 \%)$ & $21(58 \%)$ & $1(11 \%)$ & 0.02 & 1.00 \\
\hline Required dialysis (\%) & $8(20 \%)$ & $12(34 \%)$ & $16(44 \%)$ & $2(22 \%)$ & 0.13 & 0.47 \\
\hline Recovered from dialysis $(\%)^{*}$ & $4(44 \%)$ & $1(8 \%)$ & $3(19 \%)$ & $1(50 \%)$ & 0.16 & 0.61 \\
\hline Creatinine at 90 days $(\mathrm{mg} / \mathrm{dL})^{* *}$ & $1.0(0.8,1.3)$ & $1.5(1.2,2.0)$ & $1.0(0.8,1.5)$ & $1.6(1.2,2.1)$ & 0.01 & 0.02 \\
\hline Received liver transplant (\%) & $6(15 \%)$ & $9(26 \%)$ & $5(15 \%)$ & $1(11 \%)$ & 0.44 & 0.24 \\
\hline
\end{tabular}

Continuous variables presented as median (quartile 1, quartile 3 ).

PRA: prerenal azotemia, HRS: hepatorenal syndrome, ATN: acute tubular necrosis, MELD: Model for End-Stage Liver Disease, and CLIF-SOFA: Chronic Liver Failure-Sequential Organ Failure Assessment.

*Among those who required dialysis ( $n=9$ for PRA, $n=12$ for HRS, $n=16$ for ATN, and $n=2$ for other).

${ }^{* *}$ Among those who were alive and were not requiring dialysis at 90 days ( $n=24$ for PRA, $n=13$ for HRS, $n=10$ for ATN, and $n=6$ for other).

who died across all four subgroups $(p=0.84)$. Those with HRS were more likely to have a urine sodium less than $10 \mathrm{mmol} / \mathrm{L}$ compared to those with ATN (21/28 [75\%] versus $5 / 19$ [26\%]; $p=0.01$ ).

A Kaplan-Meier curve depicts survival through 90 days by type of AKI (PRA, HRS, and ATN) in Figure 2. Overall, there was a significant difference in survival between the three subgroups $(p=0.04)$. However, there was no difference in survival between HRS and ATN $(p=0.99)$. Mortality was significantly lower in PRA compared to HRS and ATN ( $p=$ 0.05 and $p=0.04$, resp.).

In a sensitivity analysis using a combined composite endpoint of death or liver transplant by 90 days, results were similar to the primary outcome, with a significant difference across all three subgroups $(p<0.001)$, but not between HRS and ATN $(p=0.41)$. When examining those with AKI and infection, there was no difference in 90-day mortality between groups with infection and those without infection ( $p=0.09$ between all groups; $p=0.06$ for AKI with infection versus PRA without infection; $p=0.39$ for AKI with infection versus HRS without infection; $p=0.97$ for AKI with infection versus ATN without infection).

Because 10 participants met HRS criteria but were classified as having ATN due to granular casts on urinary sediment, a sensitivity analysis was performed including them in the HRS subgroup. This resulted in 45 participants being classified as having HRS and reduced the number of those with ATN to 26. There was no change in the 90-day survival in this sensitivity analysis ( $p=0.03$ overall and $p=0.63$ for HRS versus ATN).

In multivariable Cox proportional hazard models containing terms for type of AKI, age, and a prognostic score (either MELD score [Model 1] or CLIF-SOFA score [Model 2]), there was a trend towards lower risk of death with a diagnosis of PRA compared to ATN (Model 1 HR: 0.52, 95\% CI [0.25-1.07]; $p=0.08$, and Model 2 HR: 0.55, 95\% CI [0.271.14]; $p=0.11$ ) while risk of death for HRS did not differ from ATN (Model 1 HR: 0.97, 95\% CI [0.52-1.79]; $p=0.91$, and Model 2 HR: 1.03, 95\% CI [0.55-1.93]; $p=0.93)$. Age was associated with increased risk of death (Model 1 HR: 1.05, 95\% CI [1.02-1.09]; $p=0.01$, and Model 2 HR: 1.05, 95\% CI [1.021.09]; $p=0.001)$. Higher MELD or CLIF-SOFA scores were associated with increased risk of death (Model 1 HR: 1.04, 95\%

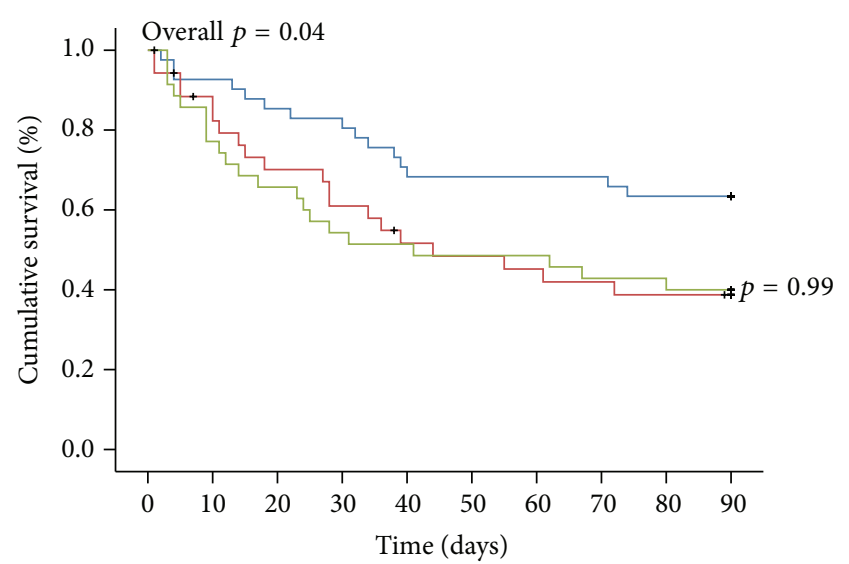

\begin{tabular}{lllll}
\hline \multicolumn{5}{l}{ Number of participants entering intervals } \\
\hline PRA & 41 & 34 & 28 & 26 \\
HRS & 35 & 20 & 14 & 11 \\
ATN & 36 & 19 & 17 & 14 \\
\hline
\end{tabular}

Type of AKI

$\rightarrow$ PRA

$\neg$ HRS

$\neg$ ATN

FIGURE 2: Ninety-day probability of survival of participants with cirrhosis by type of acute kidney injury. PRA (prerenal azotemia), HRS (hepatorenal syndrome), and ATN (acute tubular necrosis).

CI [1.01-1.08]; $p=0.04$, and Model 2 HR: 1.15, 95\% CI [1.02$1.30] ; p=0.02)$.

3.3. Hepatorenal Syndrome Diagnostic Criteria. Each of the six components of the Ascites Club Criteria was analyzed in the PRA, ATN, and "other" AKI subgroups to determine how each participant failed to meet diagnostic criteria for HRS (Figure 3). In the ATN subgroup, participants most commonly had evidence of parenchymal renal disease $(55 \%)$ or shock (33\%). In the PRA subgroup, participants most commonly had a serum creatinine lower than $1.5 \mathrm{mg} / \mathrm{dL}$ (20\%), had a reduction of creatinine below $1.5 \mathrm{mg} / \mathrm{dL}$ with albumin and holding diuretics (55\%), or had shock (20\%). In the subgroup of "other" causes of AKI, participants most commonly 


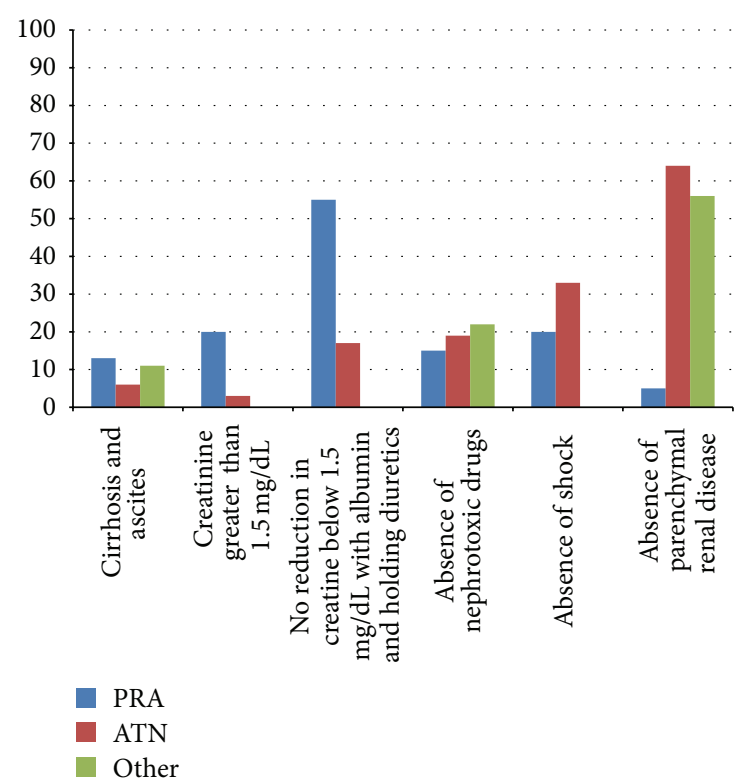

FIGURE 3: Percentage of participants with acute kidney injury who failed to satisfy Ascites Club Criteria for hepatorenal syndrome. PRA (prerenal azotemia), ATN (acute tubular necrosis).

had evidence of parenchymal renal disease $(56 \%)$ or exposure to nephrotoxic drugs (22\%).

\section{Discussion}

Our results suggest that, among those with cirrhosis and AKI, 90-day mortality is the same between those with HRS and ATN in crude analysis and after adjusting for age and a cirrhosis-specific prognostic assessment (MELD or CLIFSOFA score). Those with PRA have a better 90-day mortality rate compared to HRS and ATN. In univariate analysis, several factors related to liver and kidney function were associated with mortality, including serum creatinine, need for dialysis, urine output, international normalized ratio, and total bilirubin. Many of these factors are reflected in widely used risk scores $[8,9,26,27]$, thus highlighting their importance in prognosis and transplant allocation.

Interestingly, our results differ from the work done by Martin-Llahi and colleagues in a similar study [13]. In their single-center, prospective cohort study of inpatients with cirrhosis and AKI, these investigators found that 90-day mortality was highest in those with HRS. Those with parenchymal nephropathy had the lowest mortality, and those with hypovolemia-mediated AKI (i.e., PRA) fell in between these two groups. We do not believe that it is biologically plausible for those with PRA to have worse mortality than those with ATN. However, there may be several reasons for the differences between our studies. First, Martin-Llahi and colleagues originally classified AKI into four subgroups, including an additional category of those with AKI mediated by infection. Because infection can be a trigger of PRA (through volume depletion from fluid losses and decreased effective circulating volume), HRS (as can be the case with spontaneous bacterial peritonitis) [25, 28], and ATN (through systemic inflammation or shock) [29], we elected to include those with infection within a PRA/HRS/ATN scheme. An analysis of 90-day mortality that stratified AKI by presence of infection did not show a significant difference between groups in our cohort. Nevertheless, it may be useful to consider infection as an independent risk factor for mortality with AKI in cirrhosis $[13,30]$. Second, there are likely variations in populations between our two studies, potentially due to differences in patient demographics and local practice patterns (e.g., terlipressin, an approved treatment for HRS, is not available in the United States but is commonly employed in Europe) or in the definition of HRS and AKI (the most recent Ascites Club Criteria and AKIN definition of AKI were not available at the time of Martin-Llahi et al's study recruitment). These differences are highlighted in HRS incidences (29\% in our study versus $51 \%$ in their cohort) as well as overall 90 -day mortality (47\% versus $70 \%$, resp.). These differences, along with variable incidence and survival rates in the literature, suggest a critical need for a multicenter study to better describe the importance of type of AKI in cirrhosis.

One important area of AKI in cirrhosis is the role of the nephrologist, though to our knowledge no prior studies have examined this aspect of care. In our sample, $78 \%$ of participants received a nephrology consultation. The nephrologist can offer careful examination of the urine sediment. Coarse pigmented granular casts are a hallmark of ATN; their absence has an excellent negative predictive value in distinguishing prerenal disease (such as PRA and HRS) from intrinsic renal insults [31]. Although one criterion for HRS is the "absence of parenchymal renal injury," only significant proteinuria, hematuria, and ultrasonographic abnormalities are listed as examples [14]. The presence of granular casts is a by-product of the pathophysiology of parenchymal injury, yet it has not been listed in the diagnostic algorithm for HRS for two iterations (it was listed as "minor criteria" in the 1979 Sassari criteria) [15]. We believe that the presence of granular casts on a nephrologist's review of sediment should be considered in the diagnosis of HRS as a tool to guide therapeutic approach to this population.

While we consider HRS a diagnosis of exclusion, it remains possible that overlap between the clinical syndromes of HRS and ATN partially explains their similar mortality rates in this study. Increasingly, clinicians are recognizing the presence of bilirubin nephropathy (sometimes referred to as cholemic nephrosis) as a consequence of advanced cirrhosis. The pathophysiology of bilirubin nephropathy is similar to that of myoglobinuria or rhabdomyolysis, where high serum bilirubin levels are directly toxic to renal tubules when filtered, resulting in a clinical picture consistent with ATN [32]. In an autopsy series in patients with jaundice, van Slambrouck et al. noted that $11 / 13$ of those with a clinical diagnosis of HRS had evidence of tubular bile casts on histologic examination of the kidney [33]. Our current understanding of HRS suggests that it is a hemodynamically driven process without intrinsic renal damage; thus it is possible that some patients with HRS have a secondary insult of ATN. The absence of a diagnostic test linked to the biology of disease and nonspecific clinical criteria do not allow the clinician to 
simultaneously diagnose a patient with ATN and HRS, even though it is possible that multiple insults may explain a patient's clinical status. Martin-Llahi et al. excluded $8 \%$ of their cohort from final analysis due to a mixed etiology of AKI, which further supports this idea [13].

Regardless of the nuances in diagnostic differences between HRS and ATN, our results confirm that those with cirrhosis and AKI represent a critically ill population. Instead of debating the current framework on which we diagnose HRS, it is more important for the clinician to determine whether there is a rapidly reversible, volume-responsive injury (such as PRA) or an insult like HRS or ATN that requires close supportive care. Small trials suggest that vasoconstrictors, albumin, and (in certain circumstances) transjugular intrahepatic shunt placement may be beneficial in HRS [34-41]. Increase in mean arterial pressure (via midodrine, octreotide, or vasopressors) has been linked to improved outcomes in HRS. Maintaining acceptable blood pressure and euvolemic volume status is within the guidelines of managing ATN as well [29]. Empiric use of available therapies, along with early and appropriate use of dialysis as a bridge to transplantation, remains the cornerstone of care for all those with AKI that is not responsive to volume repletion.

Our study should be interpreted within the context of its limitations. This was a single-center trial and may not be generalizable to the experience at other liver transplant centers. However, no multicenter studies have been published examining this subject; thus we believe that our results represent an important contribution to the literature. Similarly, our cohort was predominantly male, white race, and of nonHispanic ethnicity, limiting generalizability. However, prior studies on cirrhosis and AKI either are similarly homogenous or do not report these patient characteristics, and our results describe the first American cohort in this area. This study was unable to use urine output as part of the AKIN criteria due to unreliable recorded data, a limitation that is commonly reported across many different clinical and research settings. Given our sample size, we were limited in methods of constructing a multivariable model to explain 90-day mortality and were unable to perform a model building process that allowed for screening or selection of a list of candidate variables. However, we feel that our model reflects a pragmatic scientific approach, as age and prognostic assessments like MELD and CLIF-SOFA scores have been well established as important contributors to mortality.

\section{Conclusions}

We present one of the only studies of US cohort examining implications of the etiology of AKI in cirrhosis. We showed a similar 90-day mortality rate between individuals with HRS and ATN, which was higher compared to the mortality in those with PRA. While there may be some differences in patient characteristics or in the diagnostic approach to HRS from center to center, it remains clear that AKI in cirrhosis portends a high mortality. Further study and multicenter clinical trials are needed to help clinicians better diagnose and improve outcomes in this critically ill group of patients.

\section{List of Abbreviations}

$\begin{array}{ll}\text { AKI: } & \text { Acute kidney injury } \\ \text { PRA: } & \text { Prerenal azotemia } \\ \text { ATN: } & \text { Acute tubular necrosis } \\ \text { HRS: } & \text { Hepatorenal syndrome } \\ \text { MELD: } & \text { Model for End-Stage Liver Disease } \\ \text { CLIF-SOFA: } & \text { Chronic Liver Failure-Sequential Organ } \\ & \text { Failure Assessment } \\ \text { AKIN: } & \text { Acute Kidney Injury Network. }\end{array}$

\section{Conflict of Interests}

The authors report no conflict of interests.

\section{Authors' Contribution}

Andrew S. Allegretti and Guillermo Ortiz are equally contributing first authors.

\section{Acknowledgments}

Andrew S. Allegretti is supported by NIH Grant 5T32DK007540-29. Sahir Kalim is supported by $\mathrm{NIH}$ Award KL2TR001100. Raymond T. Chung is supported by $\mathrm{NIH}$ Grant K24DK078772. S. Ananth Karumanchi is an investigator of the Howard Hughes Medical Institute.

\section{References}

[1] P. Ginès and R. W. Schrier, "Renal failure in cirrhosis," The New England Journal of Medicine, vol. 361, no. 13, pp. 1279-1290, 2009.

[2] P. Ginès, M. Guevara, V. Arroyo, and J. Rodés, "Hepatorenal syndrome," The Lancet, vol. 362, no. 9398, pp. 1819-1827, 2003.

[3] G. Garcia-Tsao, C. R. Parikh, and A. Viola, "Acute kidney injury in cirrhosis," Hepatology, vol. 48, no. 6, pp. 2064-2077, 2008.

[4] H. M. Wadei, M. L. Mai, N. Ahsan, and T. A. Gonwa, "Hepatorenal syndrome: pathophysiology and management," Clinical Journal of the American Society of Nephrology, vol. 1, no. 5, pp. 1066-1079, 2006.

[5] D. du Cheyron, B. Bouchet, J.-J. Parienti, M. Ramakers, and P. Charbonneau, "The attributable mortality of acute renal failure in critically ill patients with liver cirrhosis," Intensive Care Medicine, vol. 31, no. 12, pp. 1693-1699, 2005.

[6] E. Cholongitas, M. Senzolo, D. Patch, S. Shaw, J. O’Beirne, and A. K. Burroughs, "Cirrhotics admitted to intensive care unit: the impact of acute renal failure on mortality," European Journal of Gastroenterology and Hepatology, vol. 21, no. 7, pp. 744-750, 2009.

[7] C. D. Tsien, R. Rabie, and F. Wong, "Acute kidney injury in decompensated cirrhosis," Gut, vol. 62, no. 1, pp. 131-137, 2013.

[8] R. Wiesner, E. Edwards, R. Freeman et al., "Model for end-stage liver disease (MELD) and allocation of donor livers," Gastroenterology, vol. 124, no. 1, pp. 91-96, 2003.

[9] R. Moreau, R. Jalan, P. Gines et al., "Acute-on-chronic liver failure is a distinct syndrome that develops in patients with acute decompensation of cirrhosis," Gastroenterology, vol. 144, no. 7, pp. 1426.e9-1437.e9, 2013. 
[10] M. Lee, J.-H. Lee, S. Oh et al., "CLIF-SOFA scoring system accurately predicts short-term mortality in acutely decompensated patients with alcoholic cirrhosis: a retrospective analysis," Liver International, vol. 35, no. 1, pp. 46-57, 2015.

[11] A. Ginès, A. Escorsell, P. Ginès et al., "Incidence, predictive factors, and prognosis of the hepatorenal syndrome in cirrhosis with ascites," Gastroenterology, vol. 105, no. 1, pp. 229-236, 1993.

[12] C. Alessandria, O. Ozdogan, M. Guevara et al., "MELD score and clinical type predict prognosis in hepatorenal syndrome: relevance to liver transplantation," Hepatology, vol. 41, no. 6, pp. 1282-1289, 2005.

[13] M. Martin-Llahi, M. Guevara, A. Torre et al., "Prognostic importance of the cause of renal failure in patients with cirrhosis," Gastroenterology, vol. 140, no. 2, pp. 488.e4-496.e4, 2011.

[14] F. Salerno, A. Gerbes, P. Ginès, F. Wong, and V. Arroyo, "Diagnosis, prevention and treatment of hepatorenal syndrome in cirrhosis," Gut, vol. 56, no. 9, pp. 1310-1318, 2007.

[15] V. Arroyo, P. Ginès, A. L. Gerbes et al., "Definition and diagnostic criteria of refractory ascites and hepatorenal syndrome in cirrhosis," Hepatology, vol. 23, no. 1, pp. 164-176, 1996.

[16] C. Francoz, D. Prié, W. AbdelRazek et al., "Inaccuracies of creatinine and creatinine-based equations in candidates for liver transplantation with low creatinine: impact on the model for end-stage liver disease score," Liver Transplantation, vol. 16, no. 10, pp. 1169-1177, 2010.

[17] F. Wong, M. K. Nadim, J. A. Kellum et al., "Working Party proposal for a revised classification system of renal dysfunction in patients with cirrhosis," Gut, vol. 60, no. 5, pp. 702-709, 2011.

[18] F. Wong, J. G. O’Leary, K. R. Reddy et al., "New consensus definition of acute kidney injury accurately predicts 30-day mortality in patients with cirrhosis and infection," Gastroenterology, vol. 145, no. 6, pp. 1280.el-1288.e1, 2013.

[19] J. M. Belcher, G. Garcia-Tsao, A. J. Sanyal et al., "Association of AKI With mortality and complications in hospitalized patients with cirrhosis," Hepatology, vol. 57, no. 2, pp. 753-762, 2013.

[20] A. de Araujo and M. R. Alvares-da-Silva, "Akin criteria as a predictor of mortality in cirrhotic patients after spontaneous bacterial peritonitis," Annals of Hepatology, vol. 13, no. 3, pp. 390-395, 2014.

[21] "Section 2: AKI definition," Kidney International Supplements, vol. 2, no. 1, pp. 19-36, 2011.

[22] J. Altamirano, C. Fagundes, M. Dominguez et al., "Acute kidney injury is an early predictor of mortality for patients with alcoholic hepatitis," Clinical Gastroenterology and Hepatology, vol. 10, no. 1, pp. 65.e3-71.e3, 2012.

[23] C. Fagundes, R. Barreto, M. Guevara et al., "A modified acute kidney injury classification for diagnosis and risk stratification of impairment of kidney function in cirrhosis," Journal of Hepatology, vol. 59, no. 3, pp. 474-481, 2013.

[24] P. A. Harris, R. Taylor, R. Thielke, J. Payne, N. Gonzalez, and J. G. Conde, "Research electronic data capture (REDCap)a metadata-driven methodology and workflow process for providing translational research informatics support," Journal of Biomedical Informatics, vol. 42, no. 2, pp. 377-381, 2009.

[25] P. Sort, M. Navasa, V. Arroyo et al., "Effect of intravenous albumin on renal impairment and mortality in patients with cirrhosis and spontaneous bacterial peritonitis," The New England Journal of Medicine, vol. 341, no. 6, pp. 403-409, 1999.

[26] K.-H. Tu, C.-C. Jenq, M.-H. Tsai et al., "Outcome scoring systems for short-term prognosis in critically ill cirrhotic patients," Shock, vol. 36, no. 5, pp. 445-450, 2011.
[27] H.-C. Pan, C.-C. Jenq, M.-H. Tsai et al., "Risk models and scoring systems for predicting the prognosis in critically ill cirrhotic patients with acute kidney injury: a prospective validation study," PLoS ONE, vol. 7, no. 12, Article ID e51094, 2012.

[28] A. Follo, J. M. Llovet, M. Navasa et al., "Renal impairment after spontaneous bacterial peritonitis in cirrhosis: incidence, clinical course, predictive factors and prognosis," Hepatology, vol. 20, no. 6, pp. 1495-1501, 1994.

[29] J. G. Abuelo, "Normotensive ischemic acute renal failure," The New England Journal of Medicine, vol. 357, no. 8, pp. 797-805, 2007.

[30] R. Barreto, C. Fagundes, M. Guevara et al., “Type-1 hepatorenal syndrome associated with infections in cirrhosis: natural history, outcome of kidney function, and survival," Hepatology, vol. 59, no. 4, pp. 1505-1513, 2014.

[31] M. A. Perazella, S. G. Coca, M. Kanbay, U. C. Brewster, and C. R. Parikh, "Diagnostic value of urine microscopy for differential diagnosis of acute kidney injury in hospitalized patients," Clinical Journal of the American Society of Nephrology, vol. 3, no. 6, pp. 1615-1619, 2008.

[32] M. G. H. Betjes and I. Bajema, "The pathology of jaundicerelated renal insufficiency: cholemic nephrosis revisited," Journal of Nephrology, vol. 19, no. 2, pp. 229-233, 2006.

[33] C. M. van Slambrouck, F. Salem, S. M. Meehan, and A. Chang, "Bile cast nephropathy is a common pathologic finding for kidney injury associated with severe liver dysfunction," Kidney International, vol. 84, no. 1, pp. 192-197, 2013.

[34] M. Rössle and A. L. Gerbes, “TIPS for the treatment of refractory ascites, hepatorenal syndrome and hepatic hydrothorax: a critical update," Gut, vol. 59, no. 7, pp. 988-1000, 2010.

[35] L. L. Gluud, K. Christensen, E. Christensen, and A. Krag, "Terlipressin for hepatorenal syndrome," Cochrane Database of Systematic Reviews, vol. 9, Article ID CD005162, 2012.

[36] M. Martín-Llahí, M.-N. Pépin, M. Guevara et al., “Terlipressin and albumin vs albumin in patients with cirrhosis and hepatorenal syndrome: a randomized study," Gastroenterology, vol. 134, no. 5, pp. 1352-1359, 2008.

[37] J. C. Q. Velez and P. J. Nietert, “Therapeutic response to vasoconstrictors in hepatorenal syndrome parallels increase in mean arterial pressure: a pooled analysis of clinical trials," The American Journal of Kidney Diseases, vol. 58, no. 6, pp. 928-938, 2011.

[38] S. Ghosh, N. S. Choudhary, A. K. Sharma et al., "Noradrenaline vs terlipressin in the treatment of type 2 hepatorenal syndrome: a randomized pilot study," Liver International, vol. 33, no. 8, pp. 1187-1193, 2013.

[39] E. Esrailian, E. R. Pantangco, N. L. Kyulo, K.-Q. Hu, and B. A. Runyon, "Octreotide/midodrine therapy significantly improves renal function and 30-day survival in patients with type 1 hepatorenal syndrome," Digestive Diseases and Sciences, vol. 52, no. 3, pp. 742-748, 2007.

[40] E. T. Schroeder, G. H. Anderson Jr., and H. Smulyan, "Effects of a portacaval or peritoneovenous shunt on renin in the hepatorenal syndrome," Kidney International, vol. 15, no. 1, pp. 54-61, 1979.

[41] A. Castells, J. Saló, R. Planas et al., "Impact of shunt surgery for variceal bleeding in the natural history of ascites in cirrhosis: a retrospective study," Hepatology, vol. 20, no. 3, pp. 584-591, 1994. 


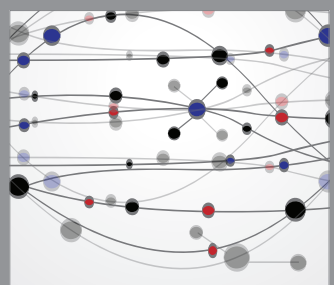

The Scientific World Journal
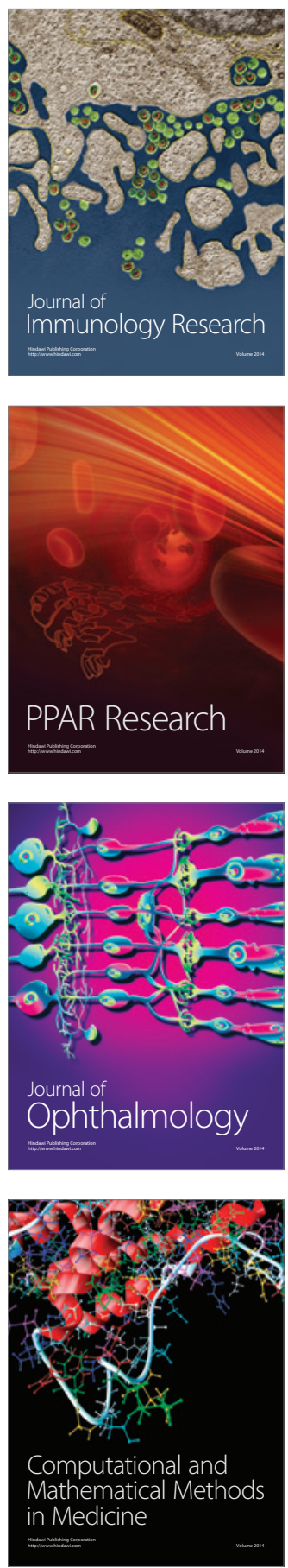

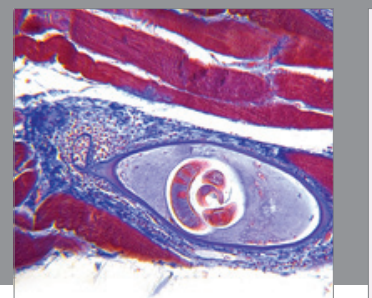

Gastroenterology

Research and Practice
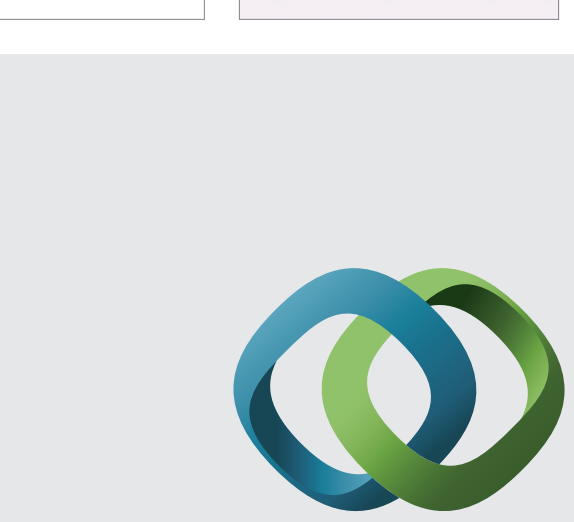

\section{Hindawi}

Submit your manuscripts at

http://www.hindawi.com
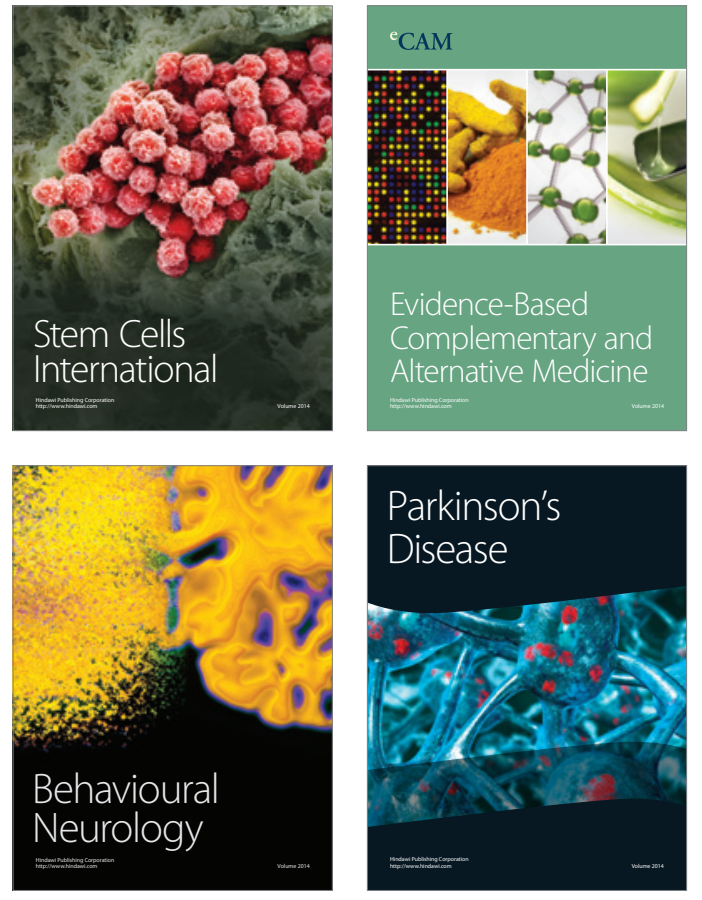
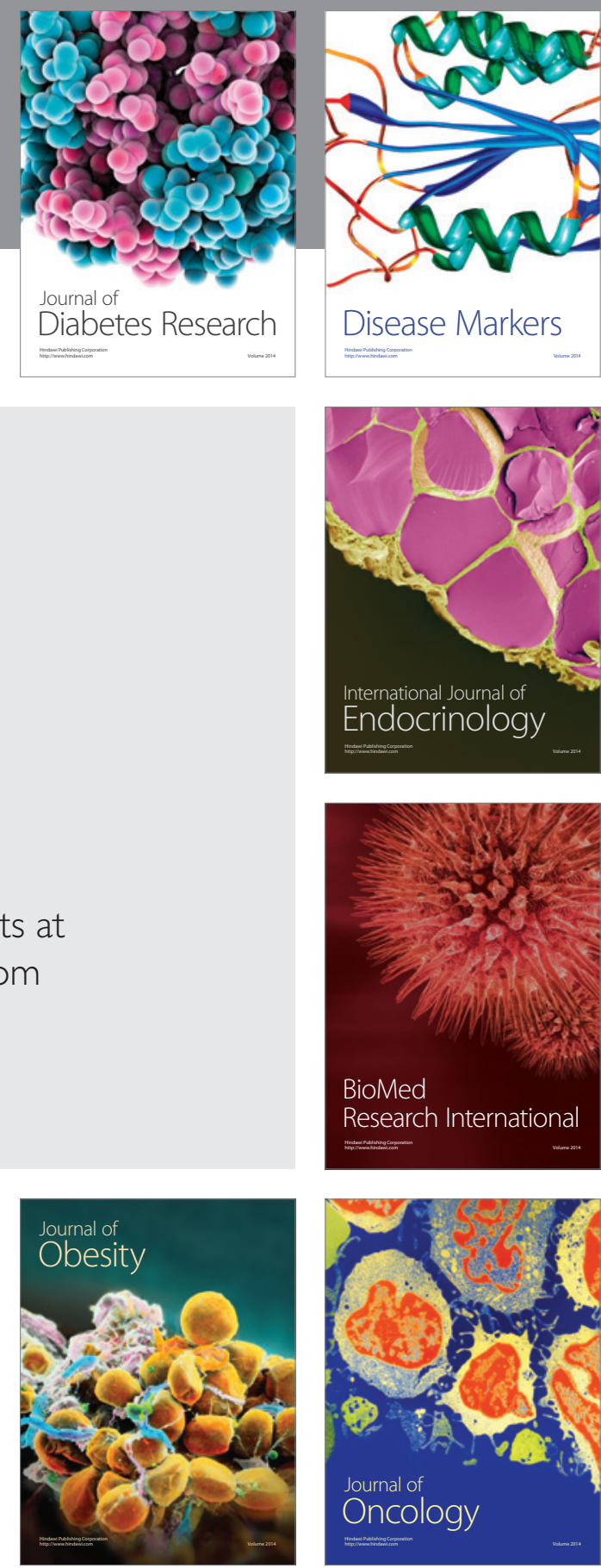

Disease Markers
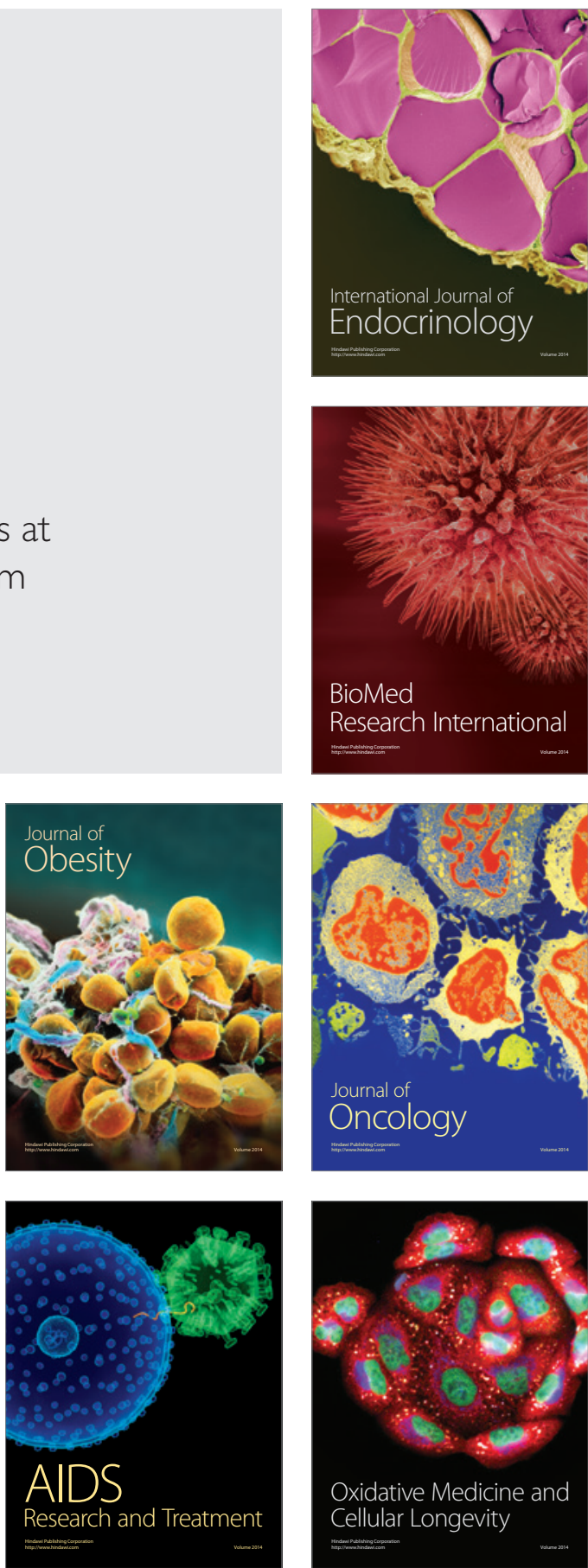